The ASTROPHYSICAL JourNaL, 303:L41-L44, 1986 April 15

(c) 1986. The American Astronomical Society. All rights reserved. Printed in U.S.A.

\title{
THE LUMINOSITY FUNCTION AND SPACE DENSITY OF THE MOST LUMINOUS GALAXIES IN THE IRAS SURVEY
}

\author{
B. T. Soifer, ${ }^{1}$ D. B. Sanders, ${ }^{1}$ G. Neugebauer, ${ }^{1}$ G. E. Danielson, ${ }^{2}$ \\ Carol J. Lonsdale, ${ }^{3}$ B. F. Madore, ${ }^{4}$ and S. E. Persson ${ }^{5}$ \\ Received 1985 December 23; accepted 1986 January 21
}

\begin{abstract}
The local space density of galaxies with $60 \mu \mathrm{m}$ luminosity greater than $\nu L_{\nu}(60 \mu \mathrm{m}) \gtrsim 10^{10} L_{\odot}$ is derived from a sample of bright galaxies detected in the IRAS survey. The sample is complete to $5 \mathrm{Jy}$ at $60 \mu \mathrm{m}$ and covers one-quarter of the sky. With a maximum redshift in the sample of 0.081 , this sample represents a survey of the infrared characteristics for infrared bright galaxies in the local universe. The space density is described by $\rho(L) \approx 1.7 \times 10^{-3}\left(L / 10^{10} L_{\odot}\right)^{-2} \mathrm{Mpc}^{-3}$ over the range $10^{10} L_{\odot}<\nu L_{\nu}(60 \mu \mathrm{m}) \leqslant 3 \times 10^{12} L_{\odot}$.

The space density of infrared bright galaxies appears comparable to or larger than other classes of extragalactic objects for total luminosities greater than $10^{11} L_{\odot}$. The space densities of infrared bright galaxies and non-Seyfert Markarian galaxies are very similar for $L<2.5 \times 10^{11} L_{\odot}$. The far-infrared energy density in the local universe is close to that in visible light. It is suggested that a substantial fraction of all galaxies with $L>10^{10} L_{\odot}$ undergo a period of extreme infrared activity.
\end{abstract}

Subject headings: galaxies: general — infrared: general - infrared: sources

\section{INTRODUCTION}

Preliminary results from the IRAS all-sky survey (de Jong et al. 1984; Soifer et al. 1984a) have shown that a significant fraction of the bolometric luminosity of galaxies emerges in the far-infrared. The far-infrared luminosity of normal spiral galaxies appears to represent $\sim 10 \%$ to $100 \%$ of the visible luminosities in these galaxies (de Jong et al. 1984), while some galaxies have been found to emit well over 100 times the energy in the far-infrared as in the visible (Soifer et al. 1984b; Aaronson and Olzewski 1984; Houck et al. 1985).

In order to understand the relative importance of infrared active galaxies compared to other classes of active extragalactic sources, and to quantify the infrared energy density of the local universe, we have undertaken a study of the brightest infrared galaxies detected in the IRAS all-sky survey. In this Letter we present the local luminosity function for galaxies with $\nu L_{\nu}(60 \mu \mathrm{m}) \geq 10^{10} L_{\odot}$. It is found that within several hundred megaparsecs the infrared luminous galaxies comprise a significant fraction of high-luminosity objects, and the infrared luminosity emitted by galaxies is a substantial fraction of that emitted in the visible portion of the spectrum.

\section{SAMPLE SELECTION AND OBSERVATIONS}

The initial sample from which the IRAS bright galaxy sample was selected consisted of all IRAS point sources (i.e., angular diameter $\Theta \leq 1^{\prime}$ ) with galactic latitude $|b| \geq 30^{\circ}$,

\footnotetext{
${ }^{1}$ Palomar Observatory California Institute of Technology.

${ }^{2}$ Palomar Observatory and Division of Geological and Planetary Sciences, California Institute of Technology.

${ }^{3}$ IPAC, California Institute of Technology.

${ }^{4}$ Division of Physics, Mathematics, and Astronomy; California Institute of Technology; and the Department of Astronomy, University of Toronto.

${ }^{5}$ Mount Wilson and Las Campanas Observatories.
}

declination $\delta \geq-20^{\circ}$, and $60 \mu \mathrm{m}$ flux densities $f_{\nu}(60 \mu \mathrm{m})$ greater than $5 \mathrm{Jy}$. After the above selection criteria were applied, sources were included in the sample by being identified with galaxies in the UGC (Nilson 1973), CGCG (Zwicky et al. 1961-1968), or Second Reference (de Vaucouleurs, de Vaucouleurs, and Corwin 1976) catalogs, by having an obvious galaxy-like optical counterpart, or by having a galaxy-like spectrum found from observations using either the double spectrograph (Oke and Gunn 1982) on the Hale $5 \mathrm{~m}$ telescope or the PFUEI (Gunn and Westphal 1981) on the Palomar $1.5 \mathrm{~m}$ telescope. Details of these observations will be given in Soifer et al. (1986). In the survey area $\left(\sim 10600 \mathrm{deg}^{2}\right.$ or one-quarter of the sky), where complete redshift information is available, there were 217 galaxies meeting the criteria. Only one infrared source had no optical counterpart on the Palomar Sky Survey, and this source was classified and discarded as being a high-latitude dark cloud, based on its infrared flux densities, its energy distribution, and the visual appearance of the field. Based on either optical spectra or associations of the infrared sample with cataloged objects, approximately $5 \%$ of the infrared sample were found to be stars or planetary nebulae, and none of the infrared sources were classified as quasars. A more detailed description of the IRAS bright galaxy sample will be given elsewhere (Soifer et al. 1986).

\section{DISCUSSION}

\section{a) Luminosities}

For some of the nearby galaxies in the sample, distance determinations were available in the literature based on either primary distance indicators (i.e., Cepheid variables) or the Tully-Fisher relation. The primary references used for 
TABLE 1

Luminosity Function of Bright GalaXies

\begin{tabular}{rccr}
\hline \hline $\begin{array}{c}\nu L_{\nu}(60 \mu \mathrm{m}) \\
\left(10^{10} L_{\odot}\right)\end{array}$ & $\begin{array}{c}\text { Space Density } \\
\left(\mathrm{Mpc}^{-3}\left[0.4 \log _{10} L / L_{\odot}\right]^{-1}\right)\end{array}$ & $V / V_{\max }$ & $N$ \\
\hline $1-2.5 \ldots \ldots$ & $5.3 \pm 0.7 \times 10^{-4}$ & $0.41 \pm 0.03$ & 53 \\
$2.5-6.3 \ldots$. & $9.7 \pm 1.6 \times 10^{-5}$ & $0.44 \pm 0.04$ & 37 \\
$6.3-16 \ldots$. & $1.1 \pm 0.3 \times 10^{-5}$ & $0.55 \pm 0.06$ & 19 \\
$16-40 \ldots \ldots$ & $4.1 \pm 0.9 \times 10^{-6}$ & $0.43 \pm 0.06$ & 21 \\
$40-100 \ldots \ldots$ & $2.6 \pm 1.1 \times 10^{-7}$ & $0.45 \pm 0.11$ & 6 \\
$100-250 \ldots \ldots$ & $7.1 \pm 3.2 \times 10^{-8}$ & $0.23 \pm 0.11$ & 5 \\
\hline
\end{tabular}

these distances were those of Aaronson and Mould (1983), Aaronson et al. (1982b), Tully and Shaya (1984), and Sandage and Tammann (1981). In the 23 cases where the distance was based on the Tully-Fisher relation, the distance was scaled to the distance adopted here for the center of the Virgo Cluster of $17.6 \mathrm{Mpc}$ (see below).

For the remaining objects, the distance was calculated using the observed redshift and the Virgocentric flow model of Aaronson et al. $(1982 a)$. In this model the combined galactic rotation and Local Group motion was taken as $V=$ $300 \sin l \cos b \mathrm{~km} \mathrm{~s}^{-1}$ ) (de Vaucouleurs, de Vaucouleurs, and Corwin 1976), and the infall velocity to Virgo was taken to be $300 \mathrm{~km} \mathrm{~s}^{-1}$. The radial velocity of Virgo was taken to be 1019 $\mathrm{km} \mathrm{s}^{-1}$, and the distance to Virgo was taken as $17.6 \mathrm{Mpc}$ (corresponding at large distances to a Hubble constant, $H_{0}=$ $75 \mathrm{~km} \mathrm{~s}^{-1} \mathrm{Mpc}^{-1}$ ).

Redshifts were taken from the literature (Palumbo, Tanzella-Nitti, and Vettolani 1983; Huchra et al. 1983) wherever possible. For those galaxies with no published redshift, the redshift was obtained from the observations on the 5 $\mathrm{m}$ or $1.5 \mathrm{~m}$ telescope. The accuracy of the redshifts determined from these later spectra is $\pm 300 \mathrm{~km} \mathrm{~s}^{-1}$. The details of these observations will be reported elsewhere (Soifer et al. 1986).

The $60 \mu \mathrm{m}$ luminosity for each galaxy was defined as $\nu L_{\nu}$ in the rest frame of the galaxy, where $\nu$ is the frequency and $L_{\nu}$ is the luminosity density. $H_{0}$ was taken as $75 \mathrm{~km} \mathrm{~s}^{-1}$ $\mathrm{Mpc}^{-1}$, and $q_{0}$ as 0 . To calculate the luminosity the flux density used was that of the IRAS Point Source Catalog, corrected for the broad-band pass of the $60 \mu \mathrm{m}$ filter, and " $K$-corrected" to the rest frame of the galaxy. With the largest redshift in the sample of $z=0.081$, and typical redshifts in the range $0.01<z<0.03$, the $K$-correction is always less than $10 \%$.

The luminosity function for this complete sample of galaxies selected at $60 \mu \mathrm{m}$ was calculated using the $V / V_{\max }$ technique (Schmidt 1968; Felton 1976). The $60 \mu \mathrm{m}$ luminosity function derived from the above data is given in Table 1 for luminosities $\nu L_{\nu}(60 \mu \mathrm{m})>10^{10} L_{\odot}$. Below this luminosity, the sample becomes more susceptible to incompleteness because some galaxies are sufficiently close that they are extended in comparison to the measuring aperture (IRAS Explanatory Supplement 1985).

\section{b) Sample Completeness and Biases}

Because the sample represents sources 10 times brighter than the completeness limit of the IRAS survey at $60 \mu \mathrm{m}$ (IRAS Explanatory Supplement 1985), the sample should be highly complete. The number of galaxies decreases with increasing $60 \mu \mathrm{m}$ flux density according to $N(>S) \propto S^{-1.5}$, consistent with a complete sample. A further test was made by calculating the mean $V / V_{\max }$ in each luminosity bin of the luminosity function. The results of these calculations are given in Table 1. Except for the lowest luminosity bin, which is affected by the Virgo Cluster, no bins show significant deviations from the expected value of 0.5 for a complete, unevolving sample. We therefore conclude that the high-luminosity portion of the IRAS Bright Galaxy Survey is statistically complete.

One obvious bias is that the selection criterion of $5 \mathrm{Jy}$ at 60 $\mu \mathrm{m}$ biases the sample against the inclusion of very cold but yet luminous galaxies. A search of the IRAS Point Source Catalog for just such a class of galaxies [i.e., high-latitude sources with spectral index $\alpha(60 \mu \mathrm{m})<-1.4$ and $\left.f_{\nu}[60 \mu]<5 \mathrm{Jy}\right]$ revealed no population of galaxies that were bright at $100 \mu \mathrm{m}$ and yet missed by the above selection criterion. Thus, while the calculations below involving total luminosity are biased toward warmer galaxies, it is clear that only a small fraction of the total population of infrared luminous galaxies have been missed.

\section{c) The Luminosity Function}

The observed luminosity function extends from $10^{10} L_{\odot}$ to greater than $10^{12} L_{\odot}$ in $60 \mu \mathrm{m}$ luminosity and is reasonably fitted by the equation $\rho \approx 1.7 \times 10^{-3}\left(L / 10^{10} L_{\odot}\right)^{-2 \pm 0.1}$ $\mathrm{Mpc}^{-3}$. The maximum luminosity sources in the bright galaxy sample have luminosities of greater than $10^{12} L_{\odot}$, well into the range of quasar luminosities (Schmidt and Green 1983), with no evidence for a cut-off at this luminosity.

The form of the luminosity function presented in Table 1 is difficult to use in comparing the space densities of different classes of extragalactic objects, since the different classes of objects emit their luminosities in widely different portions of the electromagnetic spectrum. In order to make the comparison of the IRAS bright galaxy sample with other classes of extragalactic objects, the luminosity functions have been expressed in terms of the total luminosities of the sources. For the IRAS galaxies the far-infrared luminosity has been taken as the measure of the total luminosity. The total far-infrared flux of each galaxy was calculated from the observed $60 \mu \mathrm{m}$ and $100 \mu \mathrm{m}$ flux densities using the expression

$$
F_{\text {tot }}=C(60 / 100) \times 1.26 \times\left[2.58 f_{\nu}(60 \mu \mathrm{m})+f_{\nu}(100 \mu \mathrm{m})\right] \text {. }
$$

In equation (1) $F_{\text {tot }}$ is the total flux incident above Earth; see Appendix B of the Cataloged Galaxies and Quasars Observed in the IRAS Survey 1985 for a complete description of this equation and definition of the multiplier $C(60 / 100) . F_{\text {tot }}$ is represented by a single-temperature blackbody convolved with a grain emissivity function with emissivity proportional to $\nu$ that is fitted to the observed flux density ratio at 60 and 100 $\mu \mathrm{m}$. The multiplier $C(60 / 100)$ is typically 1.5 .

The total far-infrared luminosity was derived from the total incident flux. This ignores the contribution from the $12 \mu \mathrm{m}$ and $25 \mu \mathrm{m}$ emission, which can typically amount to a $30 \%$ 
TABLE 2

FAR-INFRARED LUMINOSITY FUNCTION OF IRAS BRIGHT GALAXIES

\begin{tabular}{ccr}
\hline $\begin{array}{c}L_{\text {tot }} \\
\left(10^{10} L_{\odot}\right)\end{array}$ & $\begin{array}{c}\text { Space Density } \\
\left(\mathrm{Mpc}^{-3}\left[0.4 \log _{10} L / L_{\odot}\right]^{-1}\right)\end{array}$ & $N$ \\
\hline $1.6-4 \ldots \ldots \ldots \ldots$. & $7.6 \pm 1.1 \times 10^{-4}$ & 60 \\
$4-10 \ldots \ldots \ldots \ldots$. & $1.1 \pm 0.2 \times 10^{-4}$ & 37 \\
$10-25 \ldots \ldots \ldots \ldots$. & $1.6 \pm 0.4 \times 10^{-5}$ & 25 \\
$25-63 \ldots \ldots \ldots \ldots$. & $3.1 \pm 0.8 \times 10^{-6}$ & 19 \\
$63-160 \ldots \ldots \ldots \ldots$ & $1.7 \pm 0.7 \times 10^{-7}$ & 6 \\
$160-400 \ldots \ldots \ldots \ldots$ & $3.6 \pm 2.1 \times 10^{-8}$ & 3 \\
\hline
\end{tabular}

correction to the total luminosity. Since the basic question being addressed is the relative importance of the far-infrared emission in galaxies, we have chosen to ignore the normal stellar component in calculating the total luminosities of the IRAS galaxies. Furthermore, for the higher luminosity objects the stellar contribution becomes less important.

The far-infrared luminosity function of the bright galaxy sample is tabulated in Table 2 and plotted in Figure 1. We have compared this luminosity function with that of "normal" galaxies, starburst galaxies, Seyfert galaxies, and quasars. For normal galaxies the luminosity function was taken from Christensen (1975). The "starburst galaxy" luminosity function was taken from Huchra (1977) while that for the Markarian Seyferts (assumed to be a reasonable representation of all Seyfert galaxies) was also taken from Huchra. The local quasar luminosity function was taken from Schmidt and Green (1983).

To convert the luminosity function for "normal" galaxies to total luminosity, typical $(B-V)=0.8 \mathrm{mag}$ and bolometric correction of $0.9 \mathrm{mag}$ were assumed (i.e., the bolometric correction was consistent with the $V-K$ colors of typical galaxies: Aaronson 1977; Johnson 1966). This total luminosity effectively measures the contribution of the late-type stars that dominate the visible and near infrared light in most spiral galaxies.

For the Seyferts and quasars the correction from $\nu L_{\nu}$ (blue) to total luminosity was estimated to be a factor of 9 . To derive this, a three-step power law of the form $f_{\nu} \propto \nu^{\alpha}$ was assumed for a typical quasar/Seyfert, where the slope $\alpha$ is -1 between $3 \times 10^{12}$ and $3 \times 10^{14} \mathrm{~Hz},-0.5$ between $3 \times 10^{14}$ and $3 \times 10^{15} \mathrm{~Hz}$, and -1.5 between $3 \times 10^{15}$ and $3 \times 10^{16} \mathrm{~Hz}$ (Malkan and Sargent 1982; Malkan 1983; Elvis et al. 1986). It must be emphasized that this correction is very uncertain and must be refined when comparing space densities in detail.

The "starburst galaxies" represent a special case in this comparison, since there is a great deal of evidence (see, e.g., Rieke et al. 1980; Gehrz, Sramek, and Weedman 1983; de Jong et al. 1984; Helou, Soifer, and Rowan-Robinson 1985 ) that the great majority of infrared luminous galaxies are powered by star formation activity. We have assumed that the non-Seyfert Markarian galaxies represent the major component of the starburst galaxies (Bohuski, Fairall, and Weedman 1978). A mean $B-V$ color of $\sim 0.5 \mathrm{mag}$ and a bolometric correction of $1.2 \mathrm{mag}$ are adopted for these galaxies (Huchra 1977; Balzano 1983). This correction includes contributions for the late-type stars and for the hot stars in these galaxies. In addition, from the IRAS Point Source Catalog the median

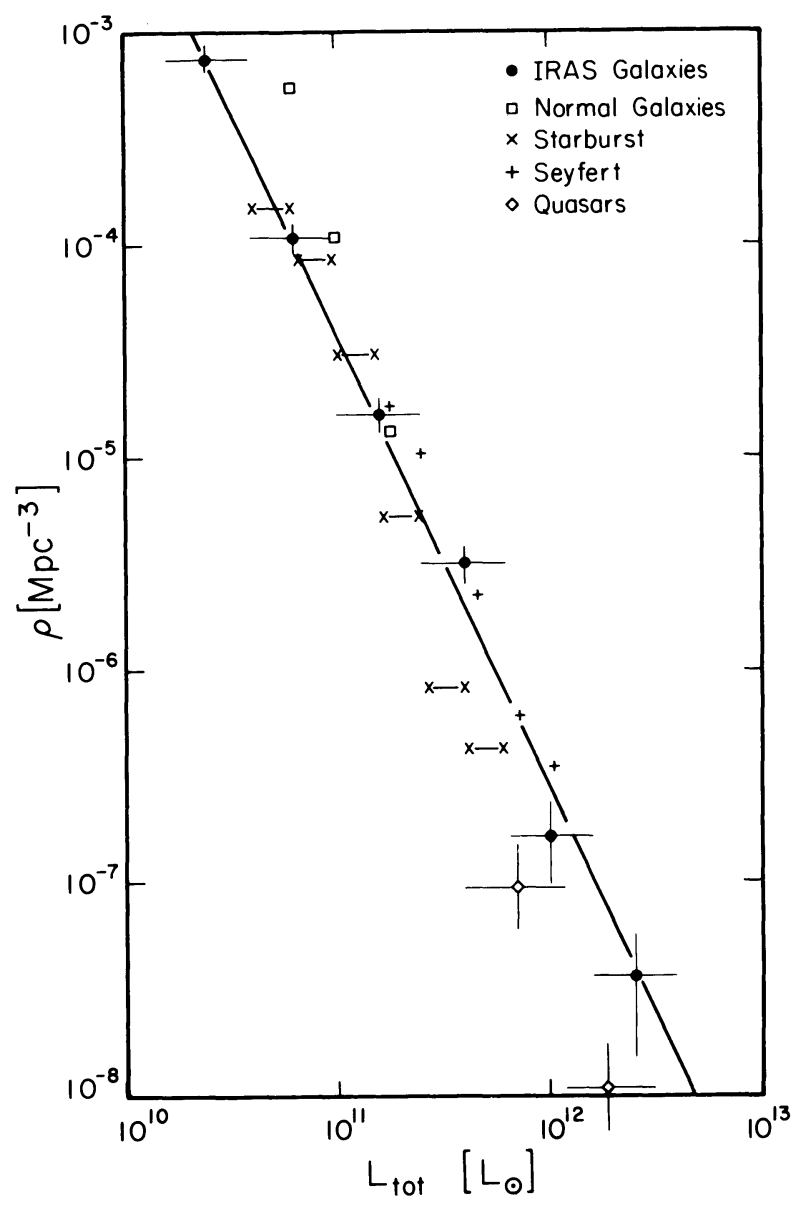

FIG. 1. - The space density of the different classes of extragalactic objects compared to those for the IRAS bright galaxy sample given in Table 2. The units of luminosity are solar luminosities, while the units of space density are galaxies per $\mathrm{Mpc}^{3}$ per magnitude interval of total luminosity. The solid line represents a crude fit to the space densities of the IRAS bright galaxies. The errors shown for the IRAS galaxies reflect the number of sources in each bin. The uncertainties in the quasar space densities represent the range of space densities in the models of Schmidt and Green (1983). The "starburst" galaxies are represented by two points at each space density, the lower luminosity representing the stellar luminosity only, while the higher luminosity contains the far-infrared contribution as described in the text.

total far-infrared luminosities of the Markarian galaxies is estimated to be 2.2 times the blue luminosity of the galaxies, or one-third of the total galaxy luminosity.

The space density of infrared galaxies in Figure 1 is quite similar to that of the starburst (non-Seyfert Markarian) galaxies at the lower luminosities, lending support to the idea that indeed these represent the same population. At the higher luminosities the bright IRAS galaxies appear more numerous than the starburst galaxies. This is suggestive either of dust affecting the UV selection of the more luminous Markarian galaxies, or possibly a new luminosity component becoming important at the highest luminosities.

Below $10^{11} L_{\odot}$ the "normal" galaxies dominate the space densities of objects in the local universe. For luminosities in excess of $2 \times 10^{11} L_{\odot}$, where the "normal" galaxy luminosity function effectively ends, the infrared luminous galaxies and 
Seyfert galaxies appear to be about equally numerous, and apparently are the most frequent classes of extragalactic objects until at least $2.5 \times 10^{12} L_{\odot}$. At the very highest luminosities, the space densities of the infrared galaxies and quasars appear to be comparable.

The total far-infrared luminosity density of the IRAS galaxies with $L_{\text {tot }}>1.6 \times 10^{10} L_{\odot}$ is $\sim 3 \times 10^{7} L_{\odot} \mathrm{Mpc}^{-3}$ as compared with a total stellar luminosity in galaxies of $\sim(1-3) \times 10^{8} L_{\odot} \mathrm{Mpc}^{-3}$ (Davis and Huchra 1982; Gott and Turner 1976; Christensen 1975). Including the contribution for the lower luminosity galaxies in the bright galaxy sample increases the far infrared luminosity density to $\sim 8 \times$ $10^{7} L_{\odot} \mathrm{Mpc}^{-3}$. This is a substantial fraction of the stellar luminosity of galaxies. Since there is strong evidence that much of the infrared luminosity in galaxies is ultimately produced by young, massive stars, the above result shows that the luminosity from such stars is a significant contribution to the total luminosity in the local universe.

Above $L_{\text {tot }}=10^{11} L_{\odot}$ the total space density of the infrared bright galaxies is $1.9 \times 10^{-5} \mathrm{Mpc}^{-3}$. Since the visible luminosities of these galaxies are $\sim 10^{10} L_{\odot}$ (Soifer et al. 1986), and this luminosity is apparently dominated by a normal stellar population, it is appropriate to compare the space densities of the infrared bright galaxies with those of the "normal" galaxies having the same visible luminosities. From Christensen (1975) the space density of "normal" galaxies with $L_{\text {tot }}>10^{10} L_{\odot}$ is $3.4 \times 10^{-3} \mathrm{Mpc}^{-3}$. Thus roughly $0.5 \%$ of galaxies with visible luminosities greater than $10^{10} L_{\odot}$ are more than 10 times more luminous in the far-infrared than in the visible. If such a stage is due to "starburst" activity having a lifetime of $\leqslant 10^{8} \mathrm{yr}$ (Rieke et al. 1980; Gehrz, Sramek, and Weedman 1983), then as many as half the galaxies with this luminosity must have experienced such an event if this is a nonrecurring stage in galaxy evolution.

We thank the staff of the Infrared Processing and Analysis Center (IPAC) for assistance and M. Schmidt and J. R. Mould for helpful discussions. B. T. S. would like to thank J. R. Houck and the Cornell University Department of Astronomy for the hospitality while the writing of this paper was begun. B. T. S., D. B. S., G. N., C. J. L., and B. F. M. were supported by NASA through the IRAS extended mission program. G. E. D. is supported by NASA contract NAS5-25451. This is contribution 4298 of the Division of Geological and Planetary Sciences.

\section{REFERENCES}

Aaronson, M. 1977, $\mathrm{Ph} . \mathrm{D}$ thesis, Harvard University.

Aaronson, M., Huchra, J., Mould, J., Schechter, P. L., and Tully, R. B. $1982 a, A p$. J., 258, 64.

Aaronson, M., and Mould, J. 1983, Ap. J., 265, 1

Aaronson, M., et al. 1982 b, Ap. J. Suppl., 50, 241.

Aaronson, M., and Olszewski, E. W. 1984, Nature, 309, 414.

Balzano, V. 1983, Ap. J., 268, 602.

Bohuski, P. J., Fairall A. P and Weedman, D. W 1978, Ap. J $221,776$.

Cataloged Galaxies and Quasars Observed in the IRAS Survey. 1985,

prepared by Carol J. Lonsdale, G. Helou, J. C. Good, and W. L. Rice

(Washington: US Government Printing Office).

Christensen, C. G. 1975, A.J., 80, 282

Davis, M., and Huchra, J. 1982, Ap. J., 254, 437.

de Jong, T., et al. 1984, A p. J. (Letters), 278, L67.

de Vaucouleurs, G., de Vaucouleurs, A., and Corwin, H. G., Jr. 1976,

Second Reference Catalog of Bright Galaxies (Austin: University of Texas Press).

Elvis, M., Green, R. F., Bechtold J., Schmidt, M., Neugebauer, G., Soifer,

B. T., Matthews, K., and Fabbiano, G. 1986, Ap. J., in press.

Felton, J. E. 1976, Ap. J., 207, 700.

Gehrz, R. D., Sramek, R. A., and Weedman, D. M. 1983, Ap. J., 267, 551.

Gott, J. R., and Turner, E. L. 1976, Ap. J., 209, 1

Gunn, J. E., and Westphal, J. A., 1981, Proc. Soc. Photo-Opt. Instr. Eng., 290, 16.

Helou, G., Soifer, B. T., and Rowan-Robinson, M. 1985, Ap. J. (Letters), 298, L7.

Houck, J. R., et al. 1985, Ap. J. (Letters), 290, L5.
Huchra, J. P. 1977, Ap. J. Suppl., 35, 171.

Huchra, J., Davis, M., Latham, D., and Tonry, J. 1983, Ap. J. Suppl., 52, 89.

IRAS Catalogs and Atlases Explanatory Supplement. 1985, ed. C. A. Beichman, G. Neugebauer, H. J. Habing, P. E. Clegg, and T. J. Chester (Washington: US Government Printing Office).

Johnson, H. L. 1966, Ann. Rev. Astr. Ap., 4, 193.

Malkan, M. 1983, Ap. J., 268, 582.

Malkan, M., and Sargent, W. L. W. 1982, Ap. J., 254, 22.

Nilson, P. 1973, Uppsala General Catalog of Galaxies, Acta Universitatis Upsaliensis, Nova Regiae Societatis Upsaliensis, Series $V: A, V o l .1$.

Palumbo, G. G. C., Tanzella-Nitta, G., and Vettolani, G. 1983, Catalog of Radial Velocities of Galaxies (New York: Gordon and Breach).

Oke, J. B., and Gunn, J. E. 1982, Pub. A.S.P., 94, 586.

Rieke, G. H., Lebofsky, M. J., Thompson, R. I., Low, F. J., and Tokunaga, A. T. 1980, Ap. J., 238, 24.

Sandage, A. R., and Tammann, G. A. 1981, A Revised Shapley-Ames Catalog of Bright Galaxies (Washington: Carnegie Institution of Washington)

Schmidt, M. 1968, Ap. J., 151, 393.

Schmidt, M., and Green, R. F. 1983, Ap. J., 269, 352.

Soifer, B. T., et al. 1984a, Ap. J. (Letters), 278, L71.

Soifer, B. T., et al. 1984b, Ap. J. (Letters), 283, L1.

Soifer, B. T., et al. 1986, in preparation.

Tully, R. B., and Shaya, E. J. 1984, Ap. J., 281, 31.

Zwicky, F., et al. 1961-1968, Catalogue of Galaxies and Clusters of Galaxies (Pasadena: California Institute of Technology), Vols. 1-6.

G. E. Danielson: California Institute of Technology, Geology and Planetary Sciences 170-25, Pasadena, CA 91125

C. J. LONSDALE: California Institute of Technology, IPAC 100-22, Pasadena, CA 91125

B. F. Madore, G. Neugebauter, D.B. Sanders, and B. T. Soifer: California Institute of Technology, Downs Building 320-47, Pasadena, CA 91125

S. E. Persson: Mount Wilson and Las Campanas Observatories, California Institute of Technology 105-24, Pasadena, CA 91125 